\title{
Interpretation of milk research results: response to comments by Michaelsson and Byberg
}

\author{
S. Sahni ${ }^{1,2}$ (1) $\cdot$ S. S. Soedamah-Muthu ${ }^{3} \cdot$ C. M. Weaver ${ }^{4}$
}

Received: 24 October 2017 / Accepted: 25 October 2017 / Published online: 16 November 2017

(C) International Osteoporosis Foundation and National Osteoporosis Foundation 2017

\section{Dear Editor,}

We appreciate the arguments raised by Michaelsson and Byberg in their letter [1]. As the authors suggest, epidemiological evidence despite a larger sample size will remain inconclusive about the effect of milk intake on the risk of mortality and fractures. In the study by Michaelsson et al. [2], we remain concerned about the sex difference comparison as the smoking rates in women were higher, the results were varying for milk vs cheese/yogurt, and for men and women, different estimates were found. The highest relative risks were in the female cohort with high milk intake (9\% of the population). These women were aged $>50$ years when the study started and were followed for 20 years. In these older women, with increasing categories of milk intake, their risk factors such as smoking as well as comorbidities also increased. Therefore, towards the end of this study, these women (aged $>70$ years) were more likely to die from multiple causes and not due to milk intake per se. Therefore, confounding remains an

S. Sahni

ShivaniSahni@hsl.harvard.edu

1 Institute for Aging Research, Hebrew SeniorLife, 1200 Centre St, Boston, MA 02131, USA

2 Department of Medicine, Beth Israel Deaconess Medical Center, Harvard Medical School, 330 Brookline Ave, Boston, MA 02215, USA

3 Center of Research on Psychology in Somatic Diseases, CoRPS, Department of Medical and Clinical Psychology, Tilburg University, P.O. Box 90153, 5000 LE Tilburg, The Netherlands

4 Department of Nutrition Science, Purdue University, STON 214, 700 W. State Street, West Lafayette, IN 2059, USA important issue in this study. We are unaware of good biomarkers for milk intake validation. In the paper by Michaelsson et al., the reference for the validation of the food frequency questionnaire (FFQ) is listed as a paper by Larsson et al. [3], which is not a validation study. Larsson et al. referred to another validation study by A. Wolk 1992, an unpublished study, which was briefly described in the Larsson paper and only provided correlations for fermented dairy and not milk. Overall, our concerns remain whether the higher risk of fractures and mortality in women reflects the true association.

\section{Compliance with ethical standards}

Conflict of interest Dr. Sahni has received institutional grants from Dairy Management Inc., and she serves as a member of the National Dairy Council's Nutrition Research Scientific Advisory Committee.

Dr. Weaver is a scientific adviser to Pharmavite, Pfizer, and Yogurt In Nutrition, Danone Institute International. Dr. Soedamah-Muthu received funding from the Global Dairy Platform, Dairy Research Institute, and Dairy Australia for a meta-analysis on cheese and blood lipids (2012) and a meta-analysis of dairy and mortality (2015). She has received the Wiebe Visser International Dairy Nutrition Prize from the Dutch Dairy Association's (NZO) Utrecht Group.

\section{References}

1. Michaëlsson K, Byberg L (2017) Interpretation of milk research results. Osteporosis Int. https://doi.org/10.1007/s00198-017-4291-x

2. Michaelsson K, Wolk A, Langenskiold S, Basu S, Warensjo Lemming E, Melhus H, Byberg L (2014) Milk intake and risk of mortality and fractures in women and men: cohort studies. BMJ 349: g6015

3. Larsson SC, Andersson SO, Johansson JE, Wolk A (2008) Cultured milk, yogurt, and dairy intake in relation to bladder cancer risk in a prospective study of Swedish women and men. Am J Clin Nutr 88(4):1083-1087 